\title{
Loi sur la psychothérapie adoptée en Allemagne
}

Peter Schulthess

La Loi de réforme sur la psychothérapie en Allemagne a été adoptée le 26 septembre 2019 et doit maintenant être mise en vigueur par le Conseil fédéral. Cela doit avoir lieu avant la fin de cette année. D’ailleurs, l'initiative de cette réforme n'est pas venue des psychothérapeutes (h/f) et de leurs associations ou des universités, mais des fonctionnaires administratifs (h/f) qui se demandaient pourquoi une profession est réglementée de telle manière qu'il faut d'abord apprendre une autre profession. En règle générale, la formation professionnelle universitaire dans les professions scientifiques est réglementée en tant que filières d'études directes. Exemples : médecin, ingénieur, architecte, avocat, pharmacien, etc. L'Allemagne avait une loi spécifique pour les psychologues et une loi spécifique pour les psychothérapeutes. Il semblait contraire au système de devoir d'abord obtenir un diplôme en psychologie avant d'être autorisé à suivre une formation en psychothérapie. Mais ensuite, après une première résistance, les universités se sont empressées d'accepter cette réforme. Les filières et cursus détudes modèles doivent être organisés et mis en place dans le cadre des facultés de psychologie ou des instituts universitaires.

Ce qui suit est un rapport du ministère fédéral allemand de la Santé daté du 27 septembre 2019 sur l'état actuel de la réforme de la Loi sur les psychothérapeutes.

\section{Peter Schulthess est membre du conseil d'administration de l'ASP.}

\section{Une formation moderne pour les psychothérapeutes}

\section{Loi portant réforme de la formation des psychothérapeutes}

La formation des psychothérapeutes (h/F) sera mise sur une nouvelle base juridique et les soins psychothérapeutiques seront améliorés. A l'avenir, le permis d'exercice en tant que psychothérapeute (h/F) sera accordé après cinq années d'études universitaires. L'accès au système de soins de l'assurance maladie obligatoire nécessite une formation postgrade ultérieure. Le nouveau cursus (filière) sera offert pour la première fois au cours du semestre d'hiver 2020.

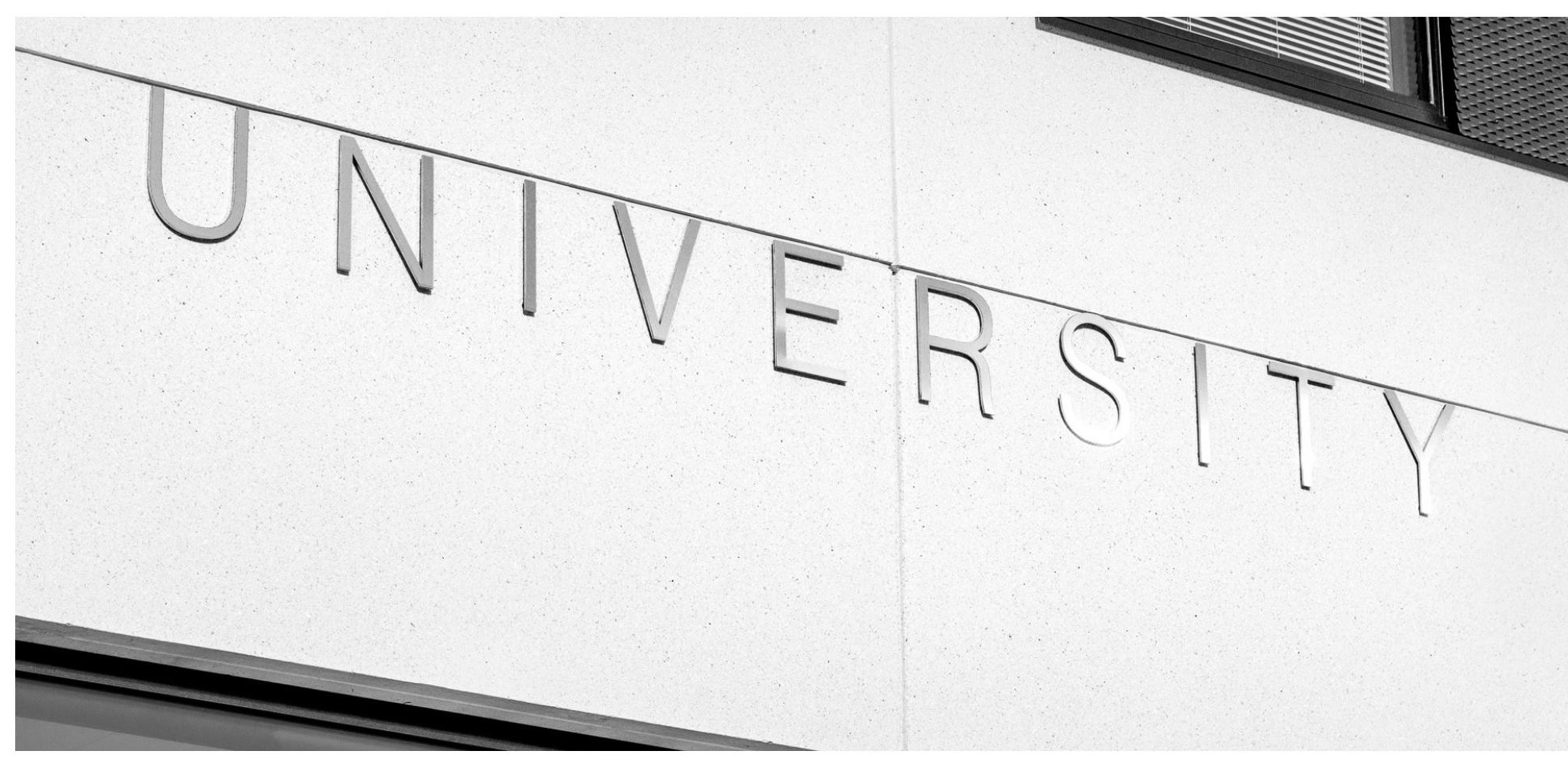




\section{Changement de la désignation professionnelle}

À l'avenir, le terme psychothérapeute (h/F) sera défini comme un titre professionnel. Jusqu'à présent, le terme a été psychothérapeute (h/F) psychologique ou psychothérapeute (h/F) d'enfants et d'adolescents. Les médecins qui offrent de la psychothérapie peuvent se dénommer des psychothérapeutes (h/F) médicaux.

\section{Une formation moderne et exigeante}

À l'avenir, les universités seront en mesure d'offrir des études directes pour la formation en psychothérapie. Ils sont divisés en une licence de trois ans de durée et une maîtrise de deux ans et se termine par un examen en tant que psychothérapeute d'État. L'approbation (permission de traiter) est accordée si l'examen est réussi.

\section{Formation postgrade : Rémunération appropriée}

Le programme d'études doit être suivi d'une formation postgrade dans des établissements hospitaliers ou ambulatoires organisés conformément à la législation nationale en vigueur. Dans le secteur ambulatoire et hospitalier, les prestations de traitement fournies par les psychothérapeutes (h/F) en formation postgrade (PiW) dans le cadre de leur formation postgrade sont remboursées par les caisses d'assurance maladie.

Une part fixe de la rémunération remboursée ou versée par les caisses d'assurance maladie aux centres de formation postgrade pour les traitements médicaux ambulatoires dispensés par les psychothérapeutes (h/F) en formation postgrade ("PiWs") doit être transmise aux PiWs. La même réglementation s'applique aux futurs psychothérapeutes (h/F) qui ont commencé leur formation selon l'ancien système ("PiAs »).

A l'avenir, les futurs psychothérapeutes (h/F) qui ont déjà commencé leur formation recevront une rémunération minimale de 1000 euros par mois pendant leur travail pratique (à plein temps) comme psychothérapeutes (h/F) en formation (" PiA »). Aucune rémunération n'a été réglementée jusqu'à présent. La rémunération minimale est refinancée par les caisses d'assurance maladie.

À l'issue de la formation postgrade, les psychothérapeutes (h/F) ont le droit de s'inscrire au registre des médecins et de demander l'admission aux soins psychothérapeutiques ambulatoires dans le système d'assurance maladie obligatoire.

\section{Améliorations dans le traitement des personnes atteintes de maladie mentale}

Le projet de loi vise également à améliorer les soins aux personnes atteintes de maladie mentale. Les médecins de famille, les psychothérapeutes (h/f), les centres de consultation en toxicomanie et les services familiaux doivent mieux coopérer à l'avenir. À cette fin, le Comité mixte fédéral doit être chargé de mieux structurer et coordonner le traitement.

La transition des soins hospitaliers aux soins ambulatoires doit également être facilitée : Les séances probatoires des psychothérapeutes (h/F) établi(e)s devraient pouvoir avoir lieu tôt dans les chambres de l'hôpital.

De plus, la participation aux thérapies de groupe ne nécessitera plus l'avis d'un expert. Cela facilite l'accès.

Afin d'inciter les psychothérapeutes (h/f) qui remplissent pleinement leur mandat de soins à offrir plus de places en thérapie, un supplément de rémunération est introduit.

La loi a été adoptée au Bundestag allemand le 26 septembre et nécessite l'approbation du Bundesrat. Elle sera annoncée au cours du second semestre 2019. 